\title{
The drug treatment of atrial fibrillation
}

\author{
K. S. CHANNER \\ Department of Cardiology, Royal Hallamshire Hospital, Glossop Road, Sheffield S10 2JF
}

1 Atrial fibrillation is an inefficient cardiac rhythm associated with impaired exercise tolerance, exertional dyspnoea, palpitation and a substantial risk of thromboembolism.

2 The first decision in management is to consider cardioversion which can be achieved in suitable cases electrically, or pharmacologically with a class Ic antiarrhythmic drug like flecainide or propafenone.

3 Prophylaxis in paroxysmal atrial fibrillation is best achieved with a class Ic drug or a class III drug such as sotalol or amiodarone.

4 Control of ventricular rate in chronic atrial fibrillation can be achieved by pharmacological manipulation of the atrioventricular node by digoxin alone, or in combination with the calcium channel blockers verapamil or diltiazem, or $\beta$-adrenoceptor blockers with intrinsic sympathomimetic activity like pindolol or xamoterol.

5 In view of the considerable risk of thromboembolism in patients with chronic atrial fibrillation anticoagulation or at least treatment with aspirin should be considered.

Keywords atrial fibrillation antiarrhythmic drugs

\section{Introduction}

Atrial fibrillation is the commonest cardiac arrhythmia. The prevalence increases with age to about $4 \%$ in patients $>74$ years (Kulbertus et al., 1984). In normal sinus rhythm the sino-atrial node positioned high in the right atrium, close to the origin of the superior vena-cava, spontaneously depolarises. The depolarisation wave front flows through the atrial muscle causing depolarisation which results in a controlled atrial contraction. When the depolarisation front reaches the atrioventricular node there is a physiological delay before it passes down the bundles of His and Purkinje fibres resulting in ventricular myocardial contraction. This electrical network provides the trigger for synchronous atrial and later ventricular contraction which optimises cardiac function.

\section{Haemodynamic effects of atrial fibrillation}

In atrial fibrillation there is chaotic atrial depolarisation which has two effects. Firstly, there is loss of atrial contraction and therefore loss of atrio-ventricular synchrony. This results in a fall in blood pressure at rest of up to $55 \%$ and cardiac output of $10-50 \%$ depending on the age of the patient and the presence of left ventricular dysfunction (Channer \& Jones, 1988). Secondly, the atrio-ventricular node is bombarded with electrical impulses at rates of between 350 and $600 \mathrm{~min}^{-1}$ from all parts of the atrium. This results in an irregular ventricular rhythm because some impulses collide with each other and are cancelled. Physiological block within the atrioventricular node relating to its effective refractory period prevents impulses of more than 180-200 beats min $^{-1}$ from being conducted to the ventricles. Nevertheless, atrial fibrillation is associated with an irregular tachycardia with wide beat to beat variation in blood pressure and cardiac output (Figure 1). It is the variability in the duration of diastole, during which the heart fills, that is responsible for these changes whilst systole remains relatively uniform.

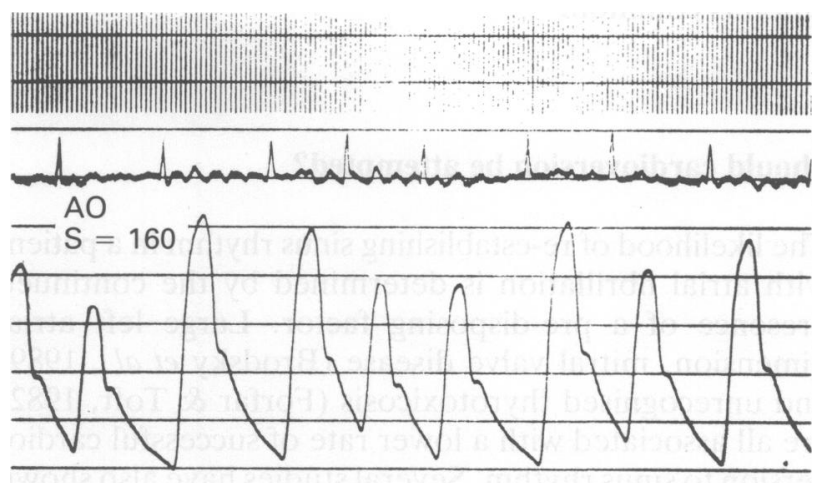

Figure 1 Aortic pressure trace showing beat to beat variation in aortic pressure in atrial fibrillation. 
The physiological consequences of atrial fibrillation are a) inefficient cardiac contraction which may result in breathlessness and impaired effort tolerance and b) an irregular tachycardia causing palpitation. Another important complication of the arrhythmia is systemic embolism. Disordered atrial contraction promotes the formation of thrombus in the atrium or atrial appendage and subsequent thromboembolism may occur. For example, in non-valvular heart disease, atrial fibrillation is associated with a five fold increase in the risk of stroke (Hinton et al., 1977; Wolf $e t$ al., 1983). There are good reasons therefore, to try to re-establish sinus rhythm in all patients with atrial fibrillation but whether this is possible depends on several factors.

\section{Pathogenesis}

It is important initially to understand the mechanism by which atrial fibrillation develops. In common with other supraventricular tachycardias a re-entrant mechanism is postulated. All myocardial cells have a refractory period during which the cell membrane is electrically silent and cannot conduct impulses. In a re-entrant mechanism adjacent cells have different refractory periods so that some can conduct whilst others are blocked. Under the right circumstances, wave fronts can circulate between areas with differing refractory periods and thus propagate the circuit. The autonomic nervous system influences the atrial refractory period and may affect some areas within the atria differently. This inhomogeneity of refractoriness pre-disposes to re-entry. One of the hallmarks of a re-entrant mechanism is the ability of a suitably timed extra stimulus to generate a tachycardia and atrial extrasystoles can initiate atrial fibrillation. Thus, in clinical terms any cause of atrial extrasystoles can potentially trigger atrial fibrillation. Ectopic activity increases with age (Rasmussen et al., 1985) as does the incidence of atrial fibrillation. Myocardial distortion causes ectopic activity (Dean \& Lab, 1989) and cardiac diseases associated with atrial dilatation and distortion are frequently associated with the development of atrial fibrillation especially mitral valve disease, hypertension and heart failure. In the presence of systemic cardiostimulants like alcohol or in thyrotoxicosis, atrial fibrillation is common.

\section{Should cardioversion be attempted?}

The likelihood of re-establishing sinus rhythm in a patient with atrial fibrillation is determined by the continued presence of a pre-disposing factor. Large left atrial dimension, mitral valve disease (Brodsky et al., 1989) and unrecognised thyrotoxicosis (Forfar \& Toft, 1982) are all associated with a lower rate of successful cardioversion to sinus rhythm. Several studies have also shown that the longer the duration of atrial fibrillation the less frequently is successful cardioversion achieved (Brodsky et al., 1989; Carr et al., 1991; Gold et al., 1986; Goy et al., 1988). The reason for this is not clear but may be related to the fact that atrial fibrillation is in itself associated with left atrial dilatation, thereby perpetuating the arrhythmia. The ideal circumstances for attempting cardioversion are therefore recent onset of atrial fibrillation and where there has been resolution or correction of any precipitating factor.

\section{Methods of cardioversion}

Cardioversion of atrial fibrillation to sinus rhythm can usually be achieved electrically by DC counter shock. This requires a general anaesthetic and therefore carries some risk, and there is now a trend to attempt cardioversion by pharmacological means initially. Given the mechanism of atrial fibrillation described above, antiarrhythmic drugs which lengthen the atrial refractory period are likely to prevent the occurrence of atrial fibrillation and may terminate it. Anti-arrhythmic drugs in the classes Ia, Ic and III have this property. Digoxin, however, which is widely used in established fibrillation to slow atrioventricular conduction, actually shortens atrial refractory period and is ineffective in preventing atrial fibrillation developing and actually prolongs the duration of bouts of atrial fibrillation (Rawles et al., 1990).

Atrial fibrillation of recent onset can be pharmacologically cardioverted with intravenous flecainide, a type Ic drug. A single intravenous bolus of $2 \mathrm{mg} \mathrm{kg}^{-1}$ given over $10 \mathrm{~min}$ will convert atrial fibrillation to sinus rhythm in between 60 and $95 \%$ of patients (Goy et al., 1988; Suttorp et al., 1989) even in those patients who are unwell after cardiac surgery (Wafa et al., 1989), acute myocardial infarction or who are currently on artificial ventilators (Carr et al., 1991). Propafenone, another type Ic drug is also effective in $60-90 \%$ of cases (Bertini et al., 1990; Bianconi et al., 1989). Amiodarone (a class III drug) given intravenously is effective in only $40-60 \%$ (Gold et al., 1986) whereas quinidine (a class Ia drug) given orally establishes sinus rhythm in about $65 \%$ of cases (Bertini et al., 1990; McAlister et al., 1990).

\section{Maintaining sinus rhythm in paroxysmal atrial fibrillation}

Once sinus rhythm has been established either by electrical cardioversion or pharmacologically, a decision should be made on the need for prophylactic treatment to prevent recurrence. When prophylaxis is required chronic oral therapy with flecainide (Anderson et al., 1989; Van Gelder et al., 1989), propafenone (Hammill et al., 1988; Kerr et al., 1988; Porterfield \& Porterfield, 1989) amiodarone (Blevins et al., 1987; Brodsky et al., 1987; Gold et al., 1986), sotalol (Antman et al., 1990) or quinidine (Hillestad et al., 1971; Sodermark et al., 1975) are effective in maintaining sinus rhythm in about 50$70 \%$ of cases when compared with placebo or no active treatment. The long term safety of these drugs in patients with paroxysmal atrial fibrillation needs to be established since all have pro-arrhythmic tendencies. Pro-arrhythmic effects of flecainide seem to be more common in older patients (Sihm et al., 1990) and in those with impaired 
left ventricular function (Brembilla-Perrot et al., 1987) and especially after an acute myocardial infarction (CAST, 1989).

\section{Chronic atrial fibrillation}

\section{Aims of treatment}

In established atrial fibrillation the aim of management is to control the ventricular rate. In the very elderly the ventricular rate may be slow enough without the need for suppressive therapy because of degenerative change in the conducting tissues. More usually the ventricular rate is fast and this is associated with short diastolic filling periods and low cardiac output. Rawles (1990) recently showed that the optimum ventricular rate at rest in atrial fibrillation was about 90 beats $\min ^{-1}$ rather than 70 beats $\min ^{-1}$ which is the optimum in sinus rhythm. He hypothesised that the higher rate was required in atrial fibrillation to compensate for the loss of effective atrial contraction. The contribution of atrial systole to left ventricular filling increases with exercise (Channer \& Jones, 1989) and Rawles (1990) predicted that the loss of atrial systole in atrial fibrillation would be compensated for by an increase in heart rate during exercise. One method of examining variations in heart rate is by using $24 \mathrm{~h}$ ambulatory electrocardiography. With this technique it is possible to obtain hourly maximum and minimum heart rates and thus plot diurnal variation (Figure 2). In patients thought clinically to have 'controlled' atrial fibrillation, ambulatory monitoring shows wide fluctuations in heart rate with $20 \%$ of patients having rates $>160 \mathrm{~min}^{-1}$ at times (Pitcher et al., 1986). Bradycardia also occurs especially at night and pauses of up to $4.0 \mathrm{~s}$ may be 'normal'. Resting heart rate in atrial fibrillation bears only a poor relation to exercise heart rate (Beasley et al., 1985) and treatment effects are better judged by ambulatory monitoring. Formal exercise testing has been used but this is hardly physiological and may bear no relation to the normal activity of the patient studied.

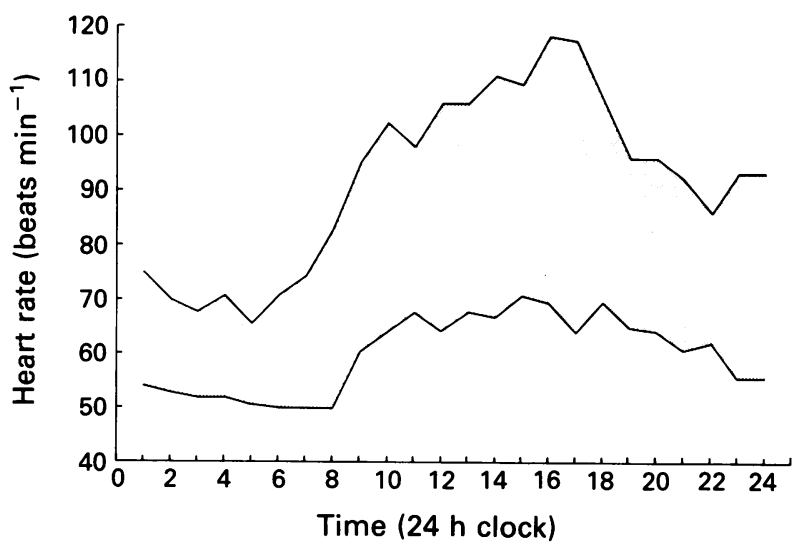

Figure 2 Plot of maximum and minimum heart rates taken from $24 \mathrm{~h}$ ambulatory heart rate monitoring of 14 patients taking high dose digoxin for atrial fibrillation.

\section{Digoxin}

Drug treatment in chronic atrial fibrillation is aimed at increasing the degree of atrio-ventricular block and thus slowing and regularising ventricular rate. Historically, digoxin has been used and is still the most widely prescribed drug in this situation. It works by inhibiting potassium/sodium dependant adenosine triphophatase. It increases vagal activity and increases the sensitivity of the sino-atrial and atrioventricular nodes to parasympathetic influences. Sympathetic activity is also reduced at therapeutic but increased at toxic doses. By modern pharmacological standards digoxin is a poor drug. About $70 \%$ is absorbed and $10 \%$ is protein bound; it is not metabolised but eliminated by the kidney. Its major problem is its narrow therapeutic index. There is marked individual variability in sensitivity in tissue responses to glycosides (Malcolm \& Coltart, 1977) and up to $20 \%$ of patients on maintenance doses have unwanted effects (Evered \& Chapman, 1971). Chamberlain (1985) wrote 'each time a glycoside is prescribed the clinician should ask whether it is worth the risk of unwanted effects, will it suit the need better than other drugs and how closely should the patient be supervised'. There are many drug interactions with digoxin and again Chamberlain (1985) urged that 'the wise physician should hesitate before prescribing a glycoside with any other drug'.

What alternative drugs are there? $\beta$-adrenoceptor blocking drugs and some calcium channel blocking drugs increase atrioventricular node refractory period and slow the ventricular response in atrial fibrillation. Unfortunately, all these drugs tend not only to be negatively chronotropic but also negatively inotropic. Since atrial fibrillation is commonly associated with heart failure, these drugs are relatively contraindicated. Digoxin however is positively inotropic and it is this fact which has allowed it to hold its place as first line treatment in chronic atrial fibrillation.

\section{Verapamil}

Verapamil was the first calcium channel blocker shown to have negative chronotropic effects on the heart. Schamroth (1971) gave it intravenously to 20 patients with atrial fibrillation (18 of whom were also taking digoxin) and showed a slowing of heart rate and a 'regularisation' of the ventricular rate. Sloman et al. (1975) showed that an intravenous bolus of verapamil given to eight digitalised patients with atrial fibrillation slowed the ventricular rate during exercise and increased stroke volume but had no effect on overall cardiac output.

Direct comparisons of chronic oral therapy with verapamil and digoxin are few and most studies have examined the effects of adding verapamil to digoxin in the control of atrial fibrillation (Schwartz et al., 1982). Verapamil ( $80 \mathrm{mg}$ three times a day) given as oral therapy is as effective as oral digoxin in lowering resting ventricular rate in patients with atrial fibrillation but is superior to digoxin in reducing exercise induced tachycardia (Lang et al., 1983; Pomfret et al., 1988). However, a reduction in diurnal maximum heart rate can also be achieved by simply doubling the dose of digoxin (Channer et al., 1987) or combining digoxin and verapamil in low dosage 
(40 mg three times daily) (Channer et al., 1987; Lewis et al., 1987; Pomfret et al., 1988). When ambulatory recordings are analysed, high dose digoxin increases nocturnal bradycardia and ventricular pauses, whereas digoxin and verapamil in combination causes less bradycardia (Channer et al., 1987). Overall heart rate variability (maximum heart rate - minimum heart rate) is thereby reduced by combining digoxin and verapamil treatment. This pharmacodynamic interaction is associated with a pharmacokinetic interaction in that the addition of oral verapamil causes a significant increase in digoxin blood level because of lowered renal clearance (Klein et al., 1982; Schwartz et al., 1982). In most clinical situations this interaction is unimportant and will not produce digoxin toxicity because most patients are taking doses of digoxin which give low serum levels-indeed arbitrarily doubling the dose failed to produce toxic symptoms in two studies (Channer et al., 1987; Lang et al., 1983) although follow up was short.

Improvements in heart rate control by verapamil with digoxin reduced palpitation scores (Channer et al., 1987; Lewis et al., 1987). Exercise tolerance however, was not consistently improved by treatment with verapamil and patients did not complain of less breathlessness when exercise induced tachycardia had been controlled (Channer et al., 1987; Lewis et al., 1988). Oxygen consumption, as a measure of work rate, has been shown to be lower in patients treated with verapamil alone and in combination with digoxin (Pomfret et al., 1988) and maximum exercise capacity has also been shown to be increased by this combination (Lang et al., 1983). However, in most studies there has been a failure to improve breathlessness and consistently increase effort tolerance in patients despite improved heart rate control. This may be explained by many factors. Firstly, the negatively inotropic effect of verapamil may offset the benefits from its negative chronotropic effects. This is unlikely to be important as the negative inotropism of verapamil is mild and studies of left ventricular ejection fraction have failed to demonstrate any effect (Pomfret et al., 1988). Secondly, as discussed earlier, the loss of effective atrial contraction in atrial fibrillation is particularly likely to be felt during exercise and although heart rate control may help it is unlikely to replace the beneficial effect of atrial systole. Thirdly, most patients with atrial fibrillation have intrinisc heart disease which may be the cause of symptoms and is unaffected by agents used to control ventricular rate.

\section{Diltiazem}

Diltiazem, another calcium antagonist, has also been shown to reduce the ventricular rate in patients with atrial fibrillation when it is used as single therapy (Lewis et al., 1988; Maragno et al., 1988). When combined with digoxin it has an additive effect (Steinberg et al., 1987) and maximum diurnal heart rate and exercise induced heart rates are lower than during treatment with either agent alone (Lewis et al., 1988; Maragno et al., 1988). At doses of $180 \mathrm{mg}$ and $240 \mathrm{mg}$ daily, it has hypotensive effects and may reduce effort tolerance in some patients because of its negative inotropic effects (Maragno et al., 1988; Roth et al., 1986). At doses of $360 \mathrm{mg}$ daily $75 \%$ of patients developed marked side-effects in one study
(Roth et al., 1986), including the development of overt heart failure.

\section{Amiodarone}

Amiodarone was originally used as an anti-anginal agent and has some calcium channel blocking properties. It has marked class III anti-arrhythmic activity and is widely used in the management of resistant ventricular tachycardia. It also lengthens the refractory period of accessory pathways and is used in Wolff-Parkinson-White syndrome. However, it lengthens the atrioventricular node refractory period and can therefore be useful in slowing the ventricular rate in atrial fibrillation. It has been used in this context (Blevins et al., 1987; Emmertsen et al., 1987) and is effective but because of its considerable side-effect profile cannot be considered to be first line therapy. One advantage it has is that there is an increased likelihood of pharmacological cardioversion from atrial fibrillation to sinus rhythm with its use.

\section{$\beta$-adrenoceptor blockers}

Another group of drugs known to increase the refractory period of the atrio-ventricular node are $\beta$-adrenoceptor blocking drugs. Intravenous treatment with propranolol (Harrison et al., 1965) in the mid 1960s clearly demonstrated an effect on resting heart rate and a blunting of the exercise induced tachycardia in atrial fibrillation. However, oral therapy with propranolol added to digoxin in six patients with atrial fibrillation caused three to develop dyspnoea despite improved heart rate control (Brown \& Goble, 1969). In another study, nadolol a long acting $\beta$-adrenoceptor blocker given to patients already taking chronic stable doses of digoxin was shown to reduce exercise induced tachycardia and diurnal maximum and minimum heart rates (DiBianco et al., 1984). Unfortunately, it also had a significant hypotensive effect and it caused a reduction in exercise duration on a treadmill by an average of $18 \%$. Atwood et al. (1987), also showed that adjuvant therapy with the $\beta$-adrenoceptor blocker celiprolol caused a $19 \%$ reduction in exercise time in digitalised patients with atrial fibrillation despite a reduction in blood pressure and resting and exercise heart rate. Hence, the worry over the negative inotropic effects of $\beta$-adrenoceptor blockade has limited their use in patients with atrial fibrillation. Nevertheless they have been shown to be effective in controlling excessive diurnal tachycardia (David et al., 1979; Yahalom et al., 1977). Nocturnal heart rate is often low in patients with atrial fibrillation and unopposed $\beta_{1}$-adrenoceptor blockade at night will worsen nocturnal bradycardia.

\section{Pindolol}

Some $\beta$-adrenoceptor blockers have intrinsic sympathomimetic activity. This allows $\beta$-adrenoceptor blockade at times of high sympathetic drive, for example, during the day and during exercise and sympathomimetic activity supervenes when sympathetic tone is low, for example, at night. Pindolol is one such $\beta$-adrenoceptor blocker and has been shown to markedly reduce the diurnal variation in heart rate in digitalised patients with atrial fibrillation, both ameliorating diurnal tachycardia 


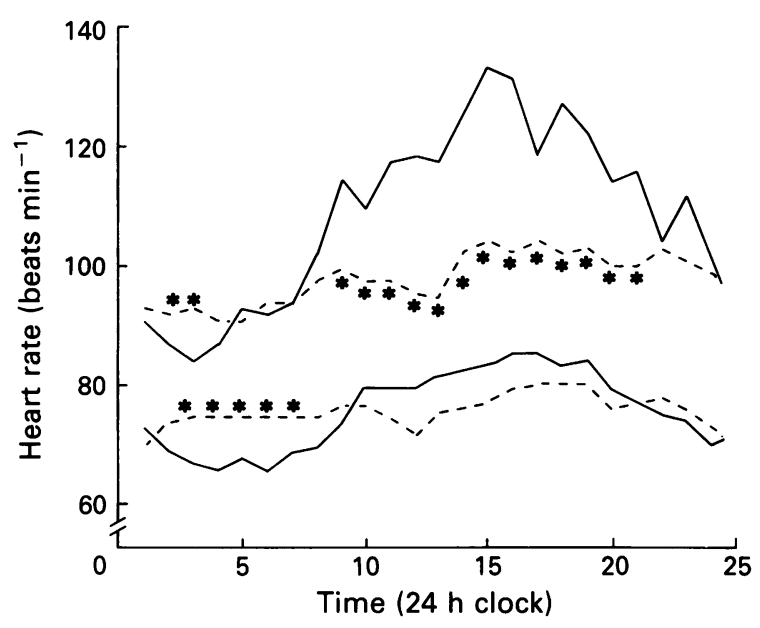

Figure 3 Plot of maximum and minimum heart rates from 10 patients taking digoxin $(-)$ or digoxin + pindolol $(15 \mathrm{mg}$ twice daily) (----) for control of atrial fibrillation. * represents significant differences. Note the virtual loss of the diurnal variation in heart rate in the pindolol treatment group. Reproduced with permission from James et al. (1989).

and preventing nocturnal bradycardia and pauses (James et al., 1989) (Figure 3). It was effective at reducing palpitation but had no effect on dyspnoea and may be less negatively inotropic than other $\beta$-adrenoceptor blockers because of its marked sympathomimetic activity. For example, in this study a quarter of the patients experienced side effects suggestive of sympathetic stimulation including feeling on edge, wakefulness, headaches and sweating. There are no pharmacokinetic interactions between $\beta$-adrenoceptor blockers and digoxin. In terms of heart rate control a combination of digoxin and pindolol would seem to be superior to digoxin \pm verapamil.

\section{Xamoterol}

Another $\beta$-adrenoceptor partial agonist, xamoterol, has also been shown to be effective at controlling ventri-

\section{References}

Anderson, J. L., Gilbert, E. M., Alpert, B. L., Henthorn, R. W., Waldo, A. L., Bhandari, A. K., Hawkinson, R. W. \& Pritchett, E. L. (1989). Prevention of symptomatic recurrences of paroxysmal atrial fibrillation in patients initially tolerating antiarrhythmic therapy. Circulation, 80, 15571570 .

Ang, E. L., Chan, W. L., Cleland, J. G. F., Moore, D., Krikler, S. J., Alexander, N. D. E. \& Oakley, C. M. (1990). Placebo controlled trial of xamoterol versus digoxin in chronic atrial fibrillation. Br. Heart J., 64, 256-260.

Antman, E. M., Beamer, A. D., Cantillon, C., McGowan, N. \& Friedman, P. L. (1990). Therapy of refractory symptomatic atrial fibrillation and atrial flutter: a staged care approach with new antiarrhythmic drugs. J. Am. Coll. Cardiol., 15, 698-707.

Atwood, J. E., Sullivan, M., Forbes, S., Myers, J., Pewen, W., Olson, H. G. \& Froelicher, V. F. (1987). Effect on beta-adrenergic blockade on exercise performance in patients with chronic atrial fibrillation. J. Am. Coll. Cardiol., 10, 314-320. cular rate in patients with atrial fibrillation (Molajo et al., 1984). Xamoterol has positive inotropic effects (James et al., 1990) and can be used in the treatment of mild heart failure (German \& Austrian Xamoterol Study, 1988), it therefore offers considerable advantages over adjuvant treatment with $\beta$-adrenoceptor blockers in atrial fibrillation. In a double-blind cross over study additional treatment with xamoterol $200 \mathrm{mg}$ twice daily to digitalised patients reduced maximum diurnal and exercise induced heart rate, increased minimal heart rate and prolonged exercise tolerance (Molajo et al., 1984). In a more recent placebo controlled study xamoterol was given to patients with atrial fibrillation and excessive bradycardia (Ang et al., 1990). Ventricular pauses and bradycardia were reduced by xamoterol compared with placebo and digoxin and exercise duration was also improved by xamoterol. Clinically xamoterol seems to be the most likely drug to replace digoxin. However, a larger randomised trial will need to be performed in far more patients to be sure of its safety and efficacy.

\section{Conclusions}

Atrial fibrillation is an inefficient cardiac rhythm and the first decision in management is to consider cardioversion. This can be achieved electrically or pharmacologically with a class Ic drug like flecainide or propafenone. Similarly, paroxysmal atrial fibrillation is best treated with a class Ic drug or a class III drug like amiodarone or sotalol. Rate control in chronic atrial fibrillation can be achieved by pharmacological manipulation of the atrioventricular node by digoxin alone or in combination with calcium channel blockers like verapamil, $\beta$-adrenoceptor blockers like pindolol or by xamoterol. Because of the considerable increase in thromboembolism in patients with chronic atrial fibrillation anticoagulation or at least treatment with aspirin should be considered in every case.
Beasley, R., Smith, D. A. \& McHaffie, D. J. (1985). Exercise heart rates at different serum digoxin concentrations in patients with atrial fibrillation. Br. med. J., 290, 9-11.

Bertini, G., Conti, A., Fradella, G., Francardelli, L., Giglioli, C., Mangialavori, G., Margheri, M. \& Moschi, G. (1990). Propafenone versus amiodarone in field treatment of primary tachydysrhythmias. J. emerg. Med., 8, 15-20.

Bianconi, L., Boccadamo, R., Pappalardo, A., Gentilli, C. \& Pistolese, M. (1989). Effectiveness of intravenous propafenone for conversion of atrial fibrillation and flutter of recent onset. Am. J. Cardiol., 64, 335-338.

Bleveins, R. D., Kerin, N. Z., Benaderet, D., Frumin, H., Faitel, K., Jarandilla, R. \& Rubenfire, M. (1987). Amiodarone in the management of refractory atrial fibrillation. Arch. intern. Med., 147, 1401-1404.

Brembilla-Perrot, B., Amor, M., Augue, F., Isaak, K., Terrier de la Chaise, A., Bertrand, A., Cherrier, F. \& Pernot, C. (1987). Effect of flecainide on left ventricular ejection fraction. Eur. Heart J., 8, 754-761.

Brodsky, M. A., Allen, B. J., Capparelli, E. V., Luckett, 
C. R., Morton, R. \& Henry, W. L. (1989). Factors determining maintenance of sinus rhythm after chronic atrial fibrillation with left atrial dilatation. Am. J. Cardiol., 63, 1065-1068.

Brodsky, M. A., Allen, B. J., Walker, C. J., Casey, T. P., Luckett, C. R. \& Henry, W. L. (1987). Amiodarone for maintenance of sinus rhythm after conversion of atrial fibrillation in the setting of a dilated left atrium. Am. J. Cardiol., 60, 572-575.

Brown, R. W. \& Goble, A. J. (1969). Effects of propranolol on exercise tolerance in patients with atrial fibrillation. $\mathrm{Br}$. med. J., 2, 279-280.

Carr, B., Hawley, K. \& Channer, K. S. (1991). Cardioversion of atrial fibrillation of recent onset with flecainide. Postgrad. med. $J .$, (in press).

CAST investigators (1989). Preliminary report: effect of encainide and flecainide on mortality in a randomised trial of arrhythmia suppression after myocardial infarction. New Engl. J. Med., 321, 406-412.

Chamberlain, D. A. (1985). Digitalis: where are we now? Br. Heart J., 54, 227-232.

Channer, K. S. \& Jones, J. V. (1988). Atrial systole: its role in normal and diseased hearts. Clin. Sci., 75, 1-4.

Channer, K. S. \& Jones, J. V. (1989). The contribution of atrial systole to mitral diastolic blood flow increases during exercise in humans. J. Physiol., 411, 53-61.

Channer, K. S., Papouchado, M., James, M. A., Pitcher, D. \& Rees, J. R. (1987). Towards improved control of atrial fibrillation. Eur. Heart J., 8, 141-147.

David, D., Segni, E. D., Klein, H. O. \& Kaplinsky, E. (1979). Inefficacy of digitalis in the control of heart rate in patients with chronic atrial fibrillation: beneficial effect on an added beta adrenergic blocking agent. Am. J. Cardiol., 44, 13781382.

Dean, J. W. \& Lab, M. J. (1989). Arrhythmia in heart failure: role of mechanically induced changes in electrophysiology. Lancet, i, 1309-1311.

DiBianco, R., Morganroth, J., Freitag, J. A., Ronan, J. A., Lindgren, K. M., Donohue, D. J., Larca, L. J., Chadda, K. D. Olukotun, A. Y. (1984). Effects of nadolol on the spontaneous and exercise-provoked heart rate of patients with chronic atrial fibrillation receiving stable dosages of digoxin. Am. Heart J., 108, 1121-1127.

Emmertsen, K., Bjerregaard, P. \& Andreasen, F. (1987). Amiodarone for refractory supraventricular tachycardias. Acta med. Scand., 221, 435-439.

Evered, D. C. \& Chapman, C. (1971). Plasma digoxin concentrations and digoxin toxicity in hospital patients. Br. Heart $J ., 33,540-550$.

Forfar, J. C. \& Toft, A. D. (1982). Thyrotoxic atrial fibrillation: an underdiagnosed condition. Br. med. J., 285, 909-910.

German and Austrian Xamoterol Study (1988). Double-blind placebo-controlled comparison of digoxin and xamoterol in chronic heart failure. Lancet, i, 489-493.

Gold, R. L., Haffajee, C. I., Charos, G., Sloan, K., Baker, S. \& Alpert, J. S. (1986). Amiodarone for refractory atrial fibrillation. Am. J. Cardiol., 57, 124-127.

Goy, J. J., Kaufman, U., Kappenberger, L. \& Sigwart, U. (1988). Restoration of sinus rhythm with flecainide in patients with atrial fibrillation. Am. J. Cardiol., 62, 38D40D.

Hammill, S. C., Wood, D. L., Gersh, B. J., Osborn, M. J. \& Holmes, D. R. (1988). Profanenone for paroxysmal atrial fibrillation. Am. J. Cardiol., 61, 473-474.

Harrison, D. C., Griffin, J. R. \& Fiene, T. J. (1965). Effects of beta adrenergic blockade with propranolol in patients with atrial fibrillation. New Engl. J. Med., 273, 410-415.

Hillestad, L., Bjerkelund, C., Dale, J., Maltau, J. \& Stortein, O. (1971). Quinidine in maintenance of sinus rhythm after electroconversion of chronic atrial fibrillation: a controlled clinical study. Br. Heart J., 33, 518-521.

Hinton, R. C., Kistler, J. P., Fallon, J. T., Freidlich, A. L. \& Fisher, C. M. (1977). Influence of etiology of atrial fibrillation on incidence of systemic embolism. Am. J. Cardiol., 40, 509-513.

James, M. A., Channer, K. S., Papouchado, M. \& Rees, J. R. (1989). Improved control of atrial fibrillation with combined pindolol and digoxin therapy. Eur. Heart J., 10, 83-90.

James, M. A., Papouchado, M., Channer, K. S., Jones, J. V., Marlow, H. F., Bastain, W., Barker, N. P., Harry, J. D. \& Wardleworth, A. G. (1990). The effect of oral dosing of xamoterol on systolic time intervals in man and xamoterol plasma concentrations in heart failure patients. Br. J. clin. Pharmac., 29, 447-453.

Kerr, C. R., Klein, G. J., Axelson, J. E. \& Cooper, J. C. (1988). Propafenone for prevention of recurrent atrial fibrillation. Am. J. Cardiol., 61, 914-916.

Klein, H. O., Lang, R., Weiss, E., DiSegni, E., Libhaber, C. \& Guerrero, J. (1982). The influence of verapamil on serum digoxin concentration. Circulation, 65, 998-1003.

Kulbertus, H. E., Leval-Rutten, F., Bartsch, P. \& Petit, J. (1984). Atrial fibrillation in elderly ambulatory patients. In Kulbertus, H. E., Olsson, A. \& Schlepper, M. eds, Atrial fibrillation, pp. 148-155. Molndal Sweden: A. B. Hassle.

Lang, R., Klein, H. O., Weiss, E., David, D., Sareli, P., Levy, A., Guerrero, J., DiSegni, E. \& Kaplinsky, E. (1983). Superiority of oral verapamil therapy to digoxin in treatment of chronic atrial fibrillation. Chest, 83, 491-499.

Lewis, R., Lakhani, M., Moreland, T. A. \& McDevitt, D. G. (1987). A comparison of verapamil and digoxin in the treatment of atrial fibrillation. Eur. Heart J., 8, 148-153.

Lewis, R. V., Irvine, N. \& McDevitt, D. G. (1988). Relationships between heart rate, exercise tolerance and cardiac output in atrial fibrillation: the effects of treatment with digoxin, verapamil and diltiazem. Eur. Heart J., 9, 777-781.

Malcolm, A. \& Coltart, J. (1977). Relation between concentrations of digoxin in the myocardium and in the plasma. Br. Heart J., 39, 935-938.

Maragno, I., Santostasi, G., Gaion, R. M., Trento, M., Grion, A. M., Miraglia, G. \& Dalla Volta, S. (1988). Low and medium dose diltiazem in chronic atrial fibrillation: comparison with digoxin and correlation with drug plasma levels. Am. Heart J., 116, 385-392.

McAlister, H. F., Luke, R. A., Whitlock, R. M. \& Smith, W. A. (1990). Intravenous amiodarone bolus versus oral quinidine for atrial flutter and fibrillation after cardiac operations. J. thoracic cardiovasc. Surg., 99, 911-918.

Molajo, A. O., Coupe, M. O. \& Bennett, D. H. (1984). Effect of Corwin (ICI 118587) on resting and exercise heart rate and exercise tolerance in digitalised patients with chronic atrial fibrillation. Br. Heart J., 52, 392-395.

Pitcher, D., Papouchado, M., James, M. A. \& Rees, J. R. (1986). Twenty four hour ambulatory electrocardiography in patients with chronic atrial fibrillation. Br. med. J., 292, 594.

Pomfret, S. M., Beasley, C. R. W., Challenor, V. \& Holgate, S. T. (1988). Relative efficacy of oral verapamil and digoxin alone and in combination for the treatment of patients with chronic atrial fibrillation. Clin. Sci., 74, 351-357.

Porterfield, J. G. \& Porterfield, L. M. (1989). Therapeutic efficacy and safety of oral propafenone for atrial fibrillation. Am. J. Cardiol., 63, 114-116.

Rasmussen, V., Jensen, G., Schnohr, P. \& Fischer Hansen, J. (1985). Premature ventricular beats in healthy adult subjects 20 to 79 years of age. Eur. Heart J., 6, 335-341.

Rawles, J. M. (1990). What is meant by a 'controlled' ventricular rate in atrial fibrillation? Br. Heart J., 63, 157-161. 
Rawles, J. M., Metcalfe, M. J. \& Jennings, K. (1990a). Time of occurrence, duration, and ventricular rate of paroxysmal atrial fibrillation: the effect of digoxin. Br. Heart J., 63, 225-227.

Roth, A., Harrison, E., Mitani, G., Cohen, J., Rahimtoola, S. \& Elkayam, U. (1986). Efficacy and safety of medium and high dose diltiazem alone and in combination with digoxin for control of heart rate at rest and during exercise in patients with atrial fibrillation. Circulation, 73, 316-324.

Schamroth, L. (1971). Immediate effects of intravenous verapamil on atrial fibrillation. Cardiovasc. Res., 5, 419-424.

Schwartz, J. B., Keefe, D., Kates, R. E., Kirsten, E. \& Harrison, D. C. (1982). Acute and chronic pharmacodynamic interaction of verapamil and digoxin in atrial fibrillation. Circulation, 65, 1163-1170.

Sihm, I., Hansen, F. A., Rasmussen, J., Pedersen, A. K. \& Thygesen, K. (1990). Flecainide acetate in atrial flutter and fibrillation. Eur. Heart J., 11, 145-148.

Sloman, G., Spokes, J., Ramshaw, J. \& Vohra, J. (1975). Haemodynamic effect of intravenous verapamil in controlled atrial fibrillation. Aust N.Z. J. Med., 5, 420-423.

Sodermark, T., Jonsson, B., Olsson, A., Oro, L., Wallin, H., Edhag, O., Sjogren, A., Danielsson, M. \& Rosenhamer, G. (1975). Effect of quinidine on maintaining sinus rhythm after conversion of atrial fibrillation or flutter: a multicentre study from Stockholm. Br. Heart J., 37, 486-492.

Steinberg, J. S., Katz, R. J., Bren, G. B., Buff, L. A. \&
Varghese, P. J. (1987). Efficacy of oral diltiazem to control ventricular response in chronic atrial fibrillation at rest and during exercise. J. Am. Coll. Cardiol., 9, 405-411.

Suttorp, M. J., Kingma, J. H., Lie-A-Huen, L. \& Mast, E. G. (1989). Intravenous flecainide versus verapamil for acute conversion of paroxysmal atrial fibrillation or flutter to sinus rhythm. Am. J. Cardiol., 63, 693-696.

Van Gelder, I., Crijns, H. J. G. M., Van Gilst, W. H., Van Wijk, L. M., Hamer, H. P. M. \& Lie, K. I. (1989). Efficacy and safety of flecaininde acetate in maintenance of sinus rhythm after electrical cardioversion of chronic atrial fibrillation or atrial flutter. Am. J. Cardiol., 64, 1317-1321.

Wafa, S. S., Ward, D. E., Parker, J. \& Camm, A. J. (1989). Efficacy of flecainide acetate for atrial arrhythmias following coronary artery bypass grafting. Am. J. Cardiol., 63, 1058-1064.

Wolf, P. A., Kannel, W. B., McGee, D. L., Meeks, S. L., Bharacha, N. E. \& McNamara, P. M. (1983). Duration of atrial fibrillation and imminence of stroke. The Framingham study. Stroke, 14, 664-667.

Yahalom, J., Klein, H. O. \& Kaplinky, E. (1977). Betaadrenergic blockade as adjunctive oral therapy in patients with chronic atrial fibrillation. Chest, 71, 592-596.

(Received 18 March 1991, accepted 27 March 1991) 